\title{
Some Coefficient Inequalities of q-Starlike Functions Associated with Conic Domain Defined by q-Derivative
}

\author{
Shahid Mahmood (D), ${ }^{1}$ Mehwish Jabeen, ${ }^{2}$ Sarfraz Nawaz Malik $\left(\mathbb{D},{ }^{2}\right.$ H. M. Srivastava (D), \\ Rabbiya Manzoor, ${ }^{2}$ and S. M. Jawwad Riaz ${ }^{2}$ \\ ${ }^{1}$ Department of Mechanical Engineering, Sarhad University of Science and IT, Ring Road, Peshawar, Pakistan \\ ${ }^{2}$ Department of Mathematics, COMSATS University Islamabad, Wah Campus, Pakistan \\ ${ }^{3}$ Department of Mathematics and Statistics, University of Victoria, Victoria, British Columbia V8W 3R4, Canada \\ ${ }^{4}$ Department of Medical Research, China Medical University Hospital, China Medical University, Taichung 40402, Taiwan
}

Correspondence should be addressed to Sarfraz Nawaz Malik; snmalik110@yahoo.com

Received 1 June 2018; Accepted 23 August 2018; Published 8 October 2018

Academic Editor: Shanhe Wu

Copyright (c) 2018 Shahid Mahmood et al. This is an open access article distributed under the Creative Commons Attribution License, which permits unrestricted use, distribution, and reproduction in any medium, provided the original work is properly cited.

This article deals with q-starlike functions associated with conic domains, defined by Janowski functions. It generalizes the recent study of q-starlike functions while associating it with the conic domains. Certain renowned coefficient inequalities in connection with the previously known ones have been included in this work.

\section{Introduction}

Quantum calculus or q-calculus is none other than a version of classical calculus because in this, we do not take limits. We consider derivatives as differences whereas antiderivatives as sums. The q-derivative of a complex valued function $f$, defined in the domain $\mathscr{D}$, is given as follows.

$$
\left(D_{q} f\right)(z)= \begin{cases}\frac{f(z)-f(q z)}{(1-q) z}, & z \neq 0, \\ f^{\prime}(0), & z=0,\end{cases}
$$

where $0<q<1$. This implies the following.

$$
\lim _{q \rightarrow 1^{-}}\left(D_{q} f\right)(z)=\lim _{q \rightarrow 1^{-}} \frac{f(z)-f(q z)}{(1-q) z}=f^{\prime}(z),
$$

provided that the function $f$ is differentiable in domain $\mathscr{D}$. The function $D_{q} f$ has Maclaurin's series representation

$$
\left(D_{q} f\right)(z)=\sum_{n=0}^{\infty}[n]_{q} a_{n} z^{n-1}
$$

where

$$
[n]_{q}= \begin{cases}\frac{1-q^{n}}{1-q}, & n \in \mathbb{C}, \\ \sum_{k=0}^{n-1} q^{k}=1+q+q^{2}+\cdots+q^{n-1}, & n \in \mathbb{N} .\end{cases}
$$

For more details about q-derivatives, we refer the reader to [1-14].

We denote by $\mathscr{A}$ the class of functions $f(z)$ which are analytic in the open unit disc $E=\{z:|z|<1\}$ and are of the form

$$
f(z)=z+\sum_{n=2}^{\infty} a_{n} z^{n}
$$

Let $S$ denote the class of all functions in $\mathscr{A}$ which are univalent in $E$. Also let $S^{*}$ and $C$ be the subclasses of $S$ consisting of all functions which map $E$ onto a star shaped with respect to origin and convex domains, respectively. A function $f$ is said to be subordinate to a function $g$, written symbolically as $f<g$, if there exists a function $w$ with $w(0)=0,|w(z)|<1$, such that $f(z)=g(w(z))$ for $z \in E$. 
The credit of systematic initiation of q-calculus goes to Jackson [13, 14] who introduced and gave the early definitions of q-derivatives and q-integrals. One of the very early contributions of usage of Q-calculus in Geometric Function Theory was made by Ismail et al. [12] who defined the generalized version of class of starlike functions. He named his newly introduced class as class of q-starlike functions since he used q-derivatives in defining it. It took a long while in further development in this direction but it proved to be a good comeback when Anastassiu and Gal $[4,5]$ presented their work on complex operators with their respective q-generalizations. These are known as qPicard and q-Gauss-Weierstrass singular integral operators. Continuing this work, Srivastava [15] laid a strong foundation of application of Q-calculus in Geometric Function Theory by using basic q-hypergeometric functions. Another series of contributions was made by Aral and Gupta [6-8] who used qbeta functions to define the q-Baskakov Durrmeyer operator. They also derived a number of geometric results with their qextensions. In the similar manner, many q-calculus operators including integral and derivative in fractional form have been used to define and analyze a number of subclasses of analytic functions. Inspired by the research carried out in q-calculus, Aldweby and Darus [1,2] defined the qoperators by using the concept of convolution of analytic functions which are normalized. Moreover, they discussed the geometrical structure of these defined operators in the analytic functions which involve q-version of hypergeometric functions in compact disc. Several useful results related to the q-version of class of close to convex functions were proved by Sahoo and Sharma [16]. Noor et al. [17] gave the research a new direction from application point of view and derived integral inequalities for relative harmonic preinvex functions. Very recently, many researchers of Geometric Function Theory like Noor et al. [18], Ramachandran et al. [19], Altinkaya et al. [3], Bulut [9], and Mahmood and Sokół [20] have contributed to the development of results in the background of q-calculus. The work on q-polynomials and $(\mathrm{p}, \mathrm{q})$-polynomials also contributed remarkably to the field of q-calculus; see $[10,11]$.

In 1999, Kanas and Wiśniowska [21] introduced the concept of conic domain by defining $k$-uniformly convex functions and then in 2000, they defined the corresponding $k$-starlike functions; see [22]. The class $k-S T$ of $k$-starlike functions is defined as follows.

A function $f(z) \in \mathscr{A}$ is said to be in the class $k-S T$ if and only if

$$
\mathfrak{R}\left[\frac{z f^{\prime}(z)}{f(z)}\right]>k\left|\frac{z f^{\prime}(z)}{f(z)}-1\right|
$$

or equivalently

$$
\frac{z f^{\prime}(z)}{f(z)} \prec \widetilde{p}_{k}(z), \quad z \in E, k \geq 0,
$$

where

$$
\tilde{p}_{k}(z)= \begin{cases}\frac{1+z}{1-z}, & k=0, \\ 1+\frac{2}{\pi^{2}}\left(\log \frac{1+\sqrt{z}}{1-\sqrt{z}}\right)^{2}, & k=1, \\ 1+\frac{2}{1-k^{2}} \sinh ^{2}\left[\left(\frac{2}{\pi} \arccos k\right) \arctan h \sqrt{z}\right], & 0<k<1, \\ 1+\frac{1}{k^{2}-1} \sin \left(\frac{\pi}{2 R(t)} \int_{0}^{u(z) / \sqrt{t}} \frac{1}{\sqrt{1-x^{2}} \sqrt{1-(t x)^{2}}} d x\right)+\frac{1}{k^{2}-1}, & k>1,\end{cases}
$$

where $u(z)=(z-\sqrt{t}) /(1-\sqrt{t z}), t \in(0,1), z \in E$, $z$ is chosen such that $k=\cosh \left(\pi R^{\prime}(t) / 4 R(t)\right), R(t)$ is the Legendre's complete elliptic integral of the first kind, and $R^{\prime}(t)$ is complementary integral of $R(t)$; for more detail, see [2125]. If $\widetilde{p}_{k}(z)=1+\delta_{k} z+\cdots$, then it is shown in [26] that from (8), one can have

$$
\delta_{k}= \begin{cases}\frac{8(\arccos k)^{2}}{\pi^{2}\left(1-k^{2}\right)}, & 0 \leq k<1, \\ \frac{8}{\pi^{2}}, & k=1, \\ \frac{\pi^{2}}{4\left(k^{2}-1\right) \sqrt{t}(1+t) R^{2}(t)}, & k>1 .\end{cases}
$$

This class $k-S T$ was then generalized by Noor and Malik [27] and its generalization $k-S T[A, B]$ was introduced by using the concept of Janowski functions. The detail of Janowski functions may be seen from [28]. The class $k-$ $S T[A, B]$ is defined as follows.

A function $f(z) \in \mathscr{A}$ is said to be in the class $k-$ $S T[A, B], k \geq 0,-1 \leq B<A \leq 1$, if and only if

$$
\begin{aligned}
& \operatorname{Re}\left(\frac{(B-1)\left(z f^{\prime}(z) / f(z)\right)-(A-1)}{(B+1)\left(z f^{\prime}(z) / f(z)\right)-(A+1)}\right) \\
& \quad>k\left|\frac{(B-1)\left(z f^{\prime}(z) / f(z)\right)-(A-1)}{(B+1)\left(z f^{\prime}(z) / f(z)\right)-(A+1)}-1\right|,
\end{aligned}
$$


or equivalently

$$
\frac{z f^{\prime}(z)}{f(z)} \prec \frac{(A+1) \tilde{p}_{k}(z)-(A-1)}{(B+1) \widetilde{p}_{k}(z)-(B-1)} .
$$

Motivated by the above classes, we now define the following more general class of q-starlike functions associated with conic domain defined by Janowski functions.

Definition 1. A function $p(z)$ is said to be in the class $k-$ $P_{q}[A, B]$, if and only if

$$
\begin{array}{r}
p(z) \\
\prec \frac{(A(1+q)+(3-q)) \tilde{p}_{k}(z)-(A(1+q)-(3-q))}{(B(1+q)+(3-q)) \widetilde{p}_{k}(z)-(B(1+q)-(3-q))}, \\
k \geq 0,
\end{array}
$$

where $\widetilde{p}_{k}(z)$ is defined by (8), $0<q<1$, and $-1 \leq B<$ $A \leq 1$.

Geometrically, the function $p(z) \in k-P[A, B]$ takes all values from the domain $\Omega_{k, q}[A, B],-1 \leq B<A \leq 1, k \geq 0$ which is defined as

$$
\begin{aligned}
\Omega_{k, q} & {[A, B]=\{w: \mathfrak{R}(\psi)>k|\psi-1|, \psi} \\
= & \left.\frac{(B(1+q)-(3-q)) w(z)-(A(1+q)-(3-q))}{(B(1+q)+(3-q)) w(z)-(A(1+q)+(3-q))}\right\} .
\end{aligned}
$$

Definition 2. A function $f(z) \in \mathscr{A}$ is said to be in the class $k-S T_{q}[A, B], k \geq 0,-1 \leq B<A \leq 1$, if and only if

$$
\begin{aligned}
\mathfrak{R}\left[\frac{(B(1+q)-(3-q))\left(z D_{q} f(z) / f(z)\right)-(A(1+q)-(3-q))}{(B(1+q)+(3-q))\left(z D_{q} f(z) / f(z)\right)-(A(1+q)+(3-q))}\right] \\
\quad>k\left|\frac{(B(1+q)-(3-q))\left(z D_{q} f(z) / f(z)\right)-(A(1+q)-(3-q))}{(B(1+q)+(3-q))\left(z D_{q} f(z) / f(z)\right)-(A(1+q)+(3-q))}-1\right|
\end{aligned}
$$

or equivalently

$$
\frac{z D_{q} f(z)}{f(z)} \in k-P_{q}[A, B] .
$$

It is noted that $0-S T_{q}[A, B]=S_{q}^{*}[A, B]$, the well-known class of q-starlike functions, introduced by Srivastava et al. [29] and $\lim _{q \longrightarrow 1-}\left(k-S T_{q}[A, B]\right)=k-S T[A, B]$, the wellknown class of Janowski k-starlike functions, introduced by Noor and Malik [27].

\section{Preliminary Results}

We need the following lemmas to prove our main results.

Lemma 3 (see [30]). Let $h(z)=1+\sum_{n=1}^{\infty} c_{n} z^{n}$ be subordinate to $H(z)=1+\sum_{n=1}^{\infty} C_{n} z^{n}$. If $H(z)$ is univalent in $E$ and $H(E)$ is convex, then

$$
\left|c_{n}\right| \leq\left|C_{1}\right|, \quad n \geq 1
$$

Lemma 4 (see [31]). If $p(z)=1+p_{1} z+p_{2} z^{2}+\cdots$ is a function with positive real part in $\mathcal{U}$, then, for any real number $v$,

$$
\left|p_{2}-v p_{1}^{2}\right| \leq \begin{cases}-4 v+2, & v \leq 0 \\ 2, & 0 \leq v \leq 1 \\ 4 v-2, & v \geq 1\end{cases}
$$

When $v<0$ or $v>1$, the equality holds if and only if $p(z)$ is $(1+z) /(1-z)$ or one of its rotations. If $0<v<1$, then, the equality holds if and only if $p(z)=\left(1+z^{2}\right) /\left(1-z^{2}\right)$ or one of its rotations. If $v=0$, the equality holds if and only if

$$
p(z)=\left(\frac{1+\eta}{2}\right) \frac{1+z}{1-z}+\left(\frac{1-\eta}{2}\right) \frac{1-z}{1+z}
$$

$$
(0 \leq \eta \leq 1) \text {, }
$$

or one of its rotations. If $v=1$, then, the equality holds if and only if $p(z)$ is reciprocal of one of the functions such that equality holds in the case of $v=0$. Although the above upper bound is sharp, when $0<v<1$, it can be improved as follows:

$$
\left|p_{2}-v p_{1}^{2}\right|+\left|p_{1}\right|^{2} \leq 2 \quad\left(0<v \leq \frac{1}{2}\right)
$$

and

$$
\left|p_{2}-v p_{1}^{2}\right|+(1-v)\left|p_{1}\right|^{2} \leq 2 \quad\left(\frac{1}{2}<v \leq 1\right) .
$$

\section{Main Results}

Theorem 5. A function $f \in A$ and of the form (5) is in the class $k-S T_{q}[A, B]$, if it satisfies the condition

$$
\begin{aligned}
\sum_{n=2}^{\infty} & \left\{2(3-q)(k+1) q[n-1]_{q}\right. \\
& +\mid(B(1+q)+(3-q))[n]_{q} \\
& -(A(1+q)+(3-q)) \mid\}\left|a_{n}\right|<(1+q)|B-A|,
\end{aligned}
$$

where $-1 \leq B<A \leq 1$ and $k \geq 0$. 


$$
\begin{aligned}
& k\left|\frac{(B(1+q)-(3-q))\left(z D_{q} f(z) / f(z)\right)-(A(1+q)-(3-q))}{(B(1+q)+(3-q))\left(z D_{q} f(z) / f(z)\right)-(A(1+q)+(3-q))}-1\right| \\
& \quad-\Re\left[\frac{(B(1+q)-(3-q))\left(z D_{q} f(z) / f(z)\right)-(A(1+q)-(3-q))}{(B(1+q)+(3-q))\left(z D_{q} f(z) / f(z)\right)-(A(1+q)+(3-q))}-1\right]<1
\end{aligned}
$$

and we consider

$$
\begin{aligned}
k \mid & \left|\frac{(B(1+q)-(3-q))\left(z D_{q} f(z) / f(z)\right)-(A(1+q)-(3-q))}{(B(1+q)+(3-q))\left(z D_{q} f(z) / f(z)\right)-(A(1+q)+(3-q))}-1\right| \\
& -\mathfrak{R}\left[\frac{(B(1+q)-(3-q))\left(z D_{q} f(z) / f(z)\right)-(A(1+q)-(3-q))}{(B(1+q)+(3-q))\left(z D_{q} f(z) / f(z)\right)-(A(1+q)+(3-q))}-1\right] \\
\leq & (k+1)\left|\frac{(B(1+q)-(3-q)) z D_{q} f(z)-(A(1+q)-(3-q)) f(z)}{(B(1+q)+(3-q)) z D_{q} f(z)-(A(1+q)+(3-q)) f(z)}-1\right| \\
& =2(3-q)(k+1)\left|\frac{f(z)-z D_{q} f(z)}{(B(1+q)+(3-q)) z D_{q} f(z)-(A(1+q)+(3-q)) f(z)}\right| \\
& =2(3-q)(k+1)\left|\frac{\sum_{n=2}^{\infty}\left(1-[n]_{q}\right) a_{n} z^{n}}{(B-A)(1+q) z+\sum_{n=2}^{\infty}\left((B(1+q)+(3-q))[n]_{q}-(A(1+q)+(3-q))\right) a_{n} z^{n}}\right| \\
& \leq \frac{2(3-q)(k+1) \sum_{n=2}^{\infty}\left|1-[n]_{q}\right|\left|a_{n}\right|}{(1+q)|B-A|-\sum_{n=2}^{\infty}\left|(B(1+q)+(3-q))[n]_{q}-(A(1+q)+(3-q))\right|\left|a_{n}\right|} .
\end{aligned}
$$

The last expression is bounded above by 1 if

$$
\begin{aligned}
2(3-q)(k+1) \sum_{n=2}^{\infty}\left([n]_{q}-1\right)\left|a_{n}\right|<(1+q)|B-A| \\
\quad-\sum_{n=2}^{\infty} \mid(B(1+q)+(3-q))[n]_{q} \\
\quad-(A(1+q)+(3-q))|| a_{n} \mid
\end{aligned}
$$

which reduces to

$$
\begin{aligned}
\sum_{n=2}^{\infty} & \left\{2(3-q)(k+1) q[n-1]_{q}\right. \\
& +\mid(B(1+q)+(3-q))[n]_{q} \\
& -(A(1+q)+(3-q)) \mid\}\left|a_{n}\right|<(1+q)|B-A|
\end{aligned}
$$

and this completes the proof.

For $q \longrightarrow 1^{-}$, Theorem 5 reduces to the following result, proved by Noor and Malik [27].
Corollary 6. A function $f \in A$ and of the form (5) is in the class $k-S T[A, B]$, if it satisfies the condition

$$
\begin{aligned}
& \sum_{n=2}^{\infty}\{2(k+1)(n-1)+|(B+1) n-(A+1)|\}\left|a_{n}\right| \\
& \quad<|B-A|,
\end{aligned}
$$

where $-1 \leq B<A \leq 1$.

Theorem 7. Let the function $f \in k-S T_{q}[A, B]$ be of the form (5); then

$$
\left|a_{n}\right| \leq \prod_{j=0}^{n-2} \frac{(A-B)(q+1) \delta_{k}-4 B q[j]_{q} \mid}{4 q[j+1]_{q}}
$$

$(n \in \mathbb{N} \backslash\{1\})$.

This result is sharp. 
Proof. By definition, for $k-S T_{q}[A, B]$, we have

$$
\frac{z D_{q} f(z)}{f(z)}=p(z)
$$

where

$$
\begin{aligned}
& p(z) \\
& \prec \frac{(A(1+q)+(3-q)) \tilde{p}_{k}(z)-(A(1+q)-(3-q))}{(B(1+q)+(3-q)) \widetilde{p}_{k}(z)-(B(1+q)-(3-q))} .
\end{aligned}
$$

If $\tilde{p}_{k}(z)=1+\delta_{k} z+\cdots$, then

$$
\begin{aligned}
& \frac{(A(1+q)+(3-q)) \tilde{p}_{k}(z)-(A(1+q)-(3-q))}{(B(1+q)+(3-q)) \widetilde{p}_{k}(z)-(B(1+q)-(3-q))} \\
& \quad=1+\frac{1}{4}(A-B)(q+1) \delta_{k} \\
& \quad+\frac{1}{4}\left[\left(-\frac{1}{4} A q-\frac{1}{4} A+\frac{1}{4} B q+\frac{1}{4} B\right)\right. \\
& \cdot((B+1)(1+q)+2-2 q)] \delta_{k}^{2}+\cdots .
\end{aligned}
$$

Now if $p(z)=1+\sum_{n=1}^{\infty} c_{n} z^{n}$, then by (16) and (29), we get

$$
\left|c_{n}\right| \leq \frac{1}{4}(A-B)(q+1)\left|\delta_{k}\right| \text {. }
$$

Now from (28), we have

$$
z D_{q} f(z)=f(z) p(z),
$$

which implies that

$$
z+\sum_{n=2}^{\infty}[n]_{q} a_{n} z^{n}=\left(z+\sum_{n=2}^{\infty} a_{n} z^{n}\right)\left(1+\sum_{n=1}^{\infty} c_{n} z^{n}\right)
$$

This implies that

$$
\sum_{n=1}^{\infty}\left([n]_{q}-1\right) a_{n} z^{n}=\sum_{n=1}^{\infty}\left(\sum_{j=1}^{n-1} a_{n-j} c_{j}\right) z^{n} .
$$

Comparison of coefficients of $z^{n}$ gives us

$$
\left([n]_{q}-1\right) a_{n}=\left(\sum_{j=1}^{n-1} a_{n-j} c_{j}\right), \quad\left(a_{1}=1\right)
$$

which reduces to

$$
\left|a_{n}\right| \leq \frac{1}{q[n-1]_{q}} \sum_{j=1}^{n-1}\left|a_{n-j}\right|\left|c_{j}\right| .
$$

By using (31), we get

$$
\left|a_{n}\right| \leq \frac{(A-B)(q+1)\left|\delta_{k}\right|}{4 q[n-1]_{q}} \sum_{j=1}^{n-1}\left|a_{j}\right| .
$$

Next, we need to show that

$$
\begin{aligned}
& \frac{(A-B)(q+1)\left|\delta_{k}\right|}{4 q[n-1]_{q}} \sum_{j=1}^{n-1}\left|a_{j}\right| \\
& \leq \prod_{j=0}^{n-2} \frac{(A-B)(q+1) \delta_{k}-4 B q[j]_{q} \mid}{4 q[j+1]_{q}} .
\end{aligned}
$$

For that, we use the principle of mathematical induction. For $n=2$, we find from (37) that

$$
\left|a_{2}\right| \leq \frac{(A-B)(q+1)\left|\delta_{k}\right|}{4 q[1]_{q}}
$$

which results also from (27). Now for $n=3$, we find from (37) that

$$
\begin{aligned}
\left|a_{3}\right| & \leq \frac{(A-B)(q+1)\left|\delta_{k}\right|}{4 q[2]_{q}}\left(1+\left|a_{2}\right|\right) \\
& \leq \frac{(A-B)(q+1)\left|\delta_{k}\right|}{4 q[2]_{q}}\left(1+\frac{(A-B)(q+1)\left|\delta_{k}\right|}{4 q[1]_{q}}\right),
\end{aligned}
$$

which also follows from (27). Now let inequality (38) be true for $n=m$. We find from (37) that

$$
\left|a_{m}\right| \leq \frac{(A-B)(q+1)\left|\delta_{k}\right|}{4 q[m-1]_{q}} \sum_{j=1}^{m-1}\left|a_{j}\right|
$$

On the other hand, from (27), we have

$$
\begin{aligned}
\left|a_{m}\right| & \leq \prod_{j=0}^{m-2} \frac{(A-B)(q+1) \delta_{k}-4 B q[j]_{q} \mid}{4 q[j+1]_{q}} \\
& \leq \prod_{j=0}^{m-2} \frac{(A-B)(q+1)\left|\delta_{k}\right|+4 q[j]_{q}}{4 q[j+1]_{q}} .
\end{aligned}
$$

By the induction hypothesis, we have

$$
\begin{aligned}
& \frac{(A-B)(q+1)\left|\delta_{k}\right|}{4 q[m-1]_{q}} \sum_{j=1}^{m-1}\left|a_{j}\right| \\
& \leq \prod_{j=0}^{m-2} \frac{(A-B)(q+1)\left|\delta_{k}\right|+4 q[j]_{q}}{4 q[j+1]_{q}} .
\end{aligned}
$$


Multiplying both sides of $(43)$ by $\left((A-B)(q+1)\left|\delta_{k}\right|+4 q[m-\right.$ $\left.1]_{q}\right) / 4 q[m]_{q}$, we have

$$
\begin{aligned}
& \prod_{j=0}^{m-1} \frac{(A-B)(q+1)\left|\delta_{k}\right|+4 q[j]_{q}}{4 q[j+1]_{q}} \\
& \geq\left(\frac{(A-B)(q+1)\left|\delta_{k}\right|+4 q[m-1]_{q}}{4 q[m]_{q}}\right) \\
& \cdot\left(\frac{(A-B)(q+1)\left|\delta_{k}\right|}{4 q[m-1]_{q}} \sum_{j=1}^{m-1}\left|a_{j}\right|\right) \\
&=\frac{(A-B)(q+1)\left|\delta_{k}\right|}{4 q[m]_{q}}\left\{\frac{(A-B)(q+1)\left|\delta_{k}\right|}{4 q[m-1]_{q}}\right. \\
&\left.\cdot \sum_{j=1}^{m-1}\left|a_{j}\right|+\sum_{j=1}^{m-1}\left|a_{j}\right|\right\} \geq \frac{(A-B)(q+1)\left|\delta_{k}\right|}{4 q[m]_{q}}\left\{\left|a_{m}\right|\right. \\
&\left.\quad+\sum_{j=1}^{m-1}\left|a_{j}\right|\right\}=\frac{(A-B)(q+1)\left|\delta_{k}\right|}{4 q[m]_{q}} \sum_{j=1}^{m}\left|a_{j}\right| .
\end{aligned}
$$

That is,

$$
\begin{aligned}
& \frac{(A-B)(q+1)\left|\delta_{k}\right|}{4 q[m]_{q}} \sum_{j=1}^{m}\left|a_{j}\right| \\
& \leq \prod_{j=0}^{m-1} \frac{(A-B)(q+1)\left|\delta_{k}\right|+4 q[j]_{q}}{4 q[j+1]}
\end{aligned}
$$

$$
\begin{aligned}
& \left|a_{3}-\mu a_{2}^{2}\right| \\
& \leq \frac{(A-B) T^{2}}{4 q\left(1-k^{2}\right)} \begin{cases}\frac{2\left(T^{2}+2\right)}{3}-\frac{((B+1)(1+q)+2(1-q)) T^{2}}{1-k^{2}}+\frac{(1+q)(A-B) T^{2}}{q\left(1-k^{2}\right)}(1-\mu(1+q)), & \mu \leq \gamma_{1}, \\
2, & \gamma_{1} \leq \mu \leq \gamma_{2}, \\
\frac{((B+1)(1+q)+2(1-q)) T^{2}}{1-k^{2}}-\frac{2\left(T^{2}+2\right)}{3}-\frac{(1+q)(A-B) T^{2}}{q\left(1-k^{2}\right)}(1-\mu(1+q)), & \mu \geq \gamma_{2},\end{cases}
\end{aligned}
$$

which shows that inequality (38) is true for $n=m+1$, and hence the required result.

For $k=0$, The above result reduces to the following result, proved by Srivastava et al. [29].

Corollary 8. Let the function $f \in S_{q}^{*}[A, B]$ be of the form (5); then

$$
\left|a_{n}\right| \leq \prod_{j=0}^{n-2} \frac{\left|(q+1)(A-B)-2 B q[j]_{q}\right|}{2 q[j+1]_{q}}
$$

$(n \in \mathbb{N} \backslash\{1\})$,

where $-1 \leq B<A \leq 1$.

For $q \longrightarrow 1^{-}$, Theorem 7 reduces to the following result, proved by Noor and Malik [27].

Corollary 9. Let the function $f \in k-S T[A, B]$ be of the form (5); then

$$
\left|a_{n}\right| \leq \prod_{j=0}^{n-2} \frac{\left|(A-B) \delta_{k}-2 B j\right|}{2(j+1)}, \quad(n \in \mathbb{N} \backslash\{1\})
$$

where $-1 \leq B<A \leq 1$.

Theorem 10. Let $f \in k-S T_{q}[A, B],-1 \leq B<A \leq 1,0<$ $k<1$ and be of the form (5). Then for real number $\mu$, we have where

$$
\begin{aligned}
\gamma_{1}= & \frac{1}{1+q}+\frac{2 q\left(T^{2}-1\right)\left(1-k^{2}\right)}{3 T^{2}(1+q)^{2}(A-B)} \\
& -\frac{q(B+1)(1+q)+2 q(1-q)}{(1+q)^{2}(A-B)}
\end{aligned}
$$

and

$$
\gamma_{2}=\frac{1}{1+q}+\frac{2 q\left(T^{2}-1\right)\left(1-k^{2}\right)}{3 T^{2}(1+q)^{2}(A-B)}
$$

$$
\begin{aligned}
& -\frac{q(B+1)(1+q)+2 q(1-q)}{(1+q)^{2}(A-B)} \\
& +\frac{4 q\left(1-k^{2}\right)}{(1+q)^{2} T^{2}(A-B)} .
\end{aligned}
$$

Proof. For $p \in P$ and of the form $p(z)=1+\sum_{n=1}^{\infty} c_{n} z^{n}$, we consider

$$
p(z)=\frac{1+w(z)}{1-w(z)}
$$


where $w(z)$ is such that $w(0)=0$ and $|w(z)|<1$. It follows easily that

$$
\begin{aligned}
w(z) & =\frac{p(z)-1}{p(z)+1} \\
& =\frac{\left(1+c_{1} z+c_{2} z^{2}+c_{3} z^{3}+\cdots\right)-1}{\left(1+c_{1} z+c_{2} z^{2}+c_{3} z^{3}+\cdots\right)+1} .
\end{aligned}
$$

This implies that

$$
\begin{aligned}
w(z)= & \frac{1}{2} c_{1} z+\left(\frac{1}{2} c_{2}-\frac{1}{4} c_{1}^{2}\right) z^{2} \\
& +\left(\frac{1}{2} c_{3}-\frac{1}{2} c_{2} c_{1}+\frac{1}{8} c_{1}^{3}\right) z^{3}+\cdots
\end{aligned}
$$

If $f(z) \in k-S T_{q}[A, B],-1 \leq B<A \leq 1,0<k<1$, then it follows from relation (15) that we have

$$
\begin{aligned}
& \frac{z D_{q} f(z)}{f(z)} \\
& <\frac{(A(1+q)+(3-q)) \widetilde{p}_{k}(z)-(A(1+q)-(3-q))}{(B(1+q)+(3-q)) \widetilde{p}_{k}(z)-(B(1+q)-(3-q))} .
\end{aligned}
$$

That is, there exists a function $w(z)$ with $w(0)=0$ and $|w(z)|<1$ such that

$$
\begin{aligned}
& \frac{z D_{q} f(z)}{f(z)} \\
& =\frac{(A(1+q)+(3-q)) \tilde{p}_{k}(w(z))-(A(1+q)-(3-q))}{(B(1+q)+(3-q)) \tilde{p}_{k}(w(z))-(B(1+q)-(3-q))},
\end{aligned}
$$

where $\tilde{p}_{k}(z)=1+\left(2 /\left(1-k^{2}\right)\right) \sinh ^{2}[((2 / \pi) \arccos k) \arctan h \sqrt{z}]$, $0<k<1$. Now if $\widetilde{p}_{k}(z)=1+R_{1}(k) z+R_{2}(k) z^{2}+R_{3}(k) z^{3}+\cdots$, then from (53), one may have

$$
\begin{aligned}
\tilde{p}_{k}(w(z))=1+R_{1}(k) w(z)+R_{2}(k) w^{2}(z)+R_{3}(k) \\
\quad w^{3}(z)+\cdots \\
=1+R_{1}(k)\left(\frac{1}{2} c_{1} z+\left(\frac{1}{2} c_{2}-\frac{1}{4} c_{1}^{2}\right) z^{2}\right. \\
\left.+\left(\frac{1}{2} c_{3}-\frac{1}{2} c_{2} c_{1}+\frac{1}{8} c_{1}^{3}\right) z^{3}+\cdots\right)+R_{2}(k)\left(\frac{1}{2} c_{1} z\right. \\
+\left(\frac{1}{2} c_{2}-\frac{1}{4} c_{1}^{2}\right) z^{2}+\left(\frac{1}{2} c_{3}-\frac{1}{2} c_{2} c_{1}+\frac{1}{8} c_{1}^{3}\right) z^{3} \\
+\cdots)^{2}+R_{3}(k)\left(\frac{1}{2} c_{1} z+\left(\frac{1}{2} c_{2}-\frac{1}{4} c_{1}^{2}\right) z^{2}\right. \\
\left.+\left(\frac{1}{2} c_{3}-\frac{1}{2} c_{2} c_{1}+\frac{1}{8} c_{1}^{3}\right) z^{3}+\cdots\right)^{3}+\cdots
\end{aligned}
$$

where $R_{1}(k), R_{2}(k)$, and $R_{3}(k)$ are given by

$$
\begin{aligned}
& R_{1}(k)=\frac{2 T^{2}}{1-k^{2}}, \\
& R_{2}(k)=\frac{2 T^{2}}{3\left(1-k^{2}\right)}\left(2+T^{2}\right), \\
& R_{3}(k)=\frac{2 T^{2}}{9\left(1-k^{2}\right)}\left(\frac{23}{5}+4 T^{2}+\frac{2}{5} T^{4}\right)
\end{aligned}
$$

and $T=T(k)=(2 / \pi) \arccos (k), 0 \leq k \leq 1$; see [26]. Using these, the above series reduces to

$$
\begin{gathered}
\widetilde{p}_{k}(w(z))=1+\frac{T^{2}}{1-k^{2}} c_{1} z+\frac{T^{2}}{1-k^{2}}\left(\left(T^{2}-1\right) \frac{1}{6} c_{1}^{2}\right. \\
\left.+c_{2}\right) z^{2}+\frac{T^{2}}{1-k^{2}}\left(\frac{1}{9}\left(\frac{2}{5}-\frac{1}{2} T^{2}+\frac{1}{10} T^{4}\right) c_{1}^{3}\right. \\
\left.-\frac{1}{3}\left(1-T^{2}\right) c_{2} c_{1}+c_{3}\right) z^{3}+\cdots .
\end{gathered}
$$

Using this, (55) becomes

$$
\begin{aligned}
& \frac{z D_{q} f(z)}{f(z)}=1+\left(\frac{(A-B)(1+q) T^{2} c_{1}}{4\left(1-k^{2}\right)}\right) z \\
& +\left[\frac { ( A - B ) ( 1 + q ) T ^ { 2 } } { 8 ( 1 - k ^ { 2 } ) } \left(\frac{\left(T^{2}-1\right)}{3}\right.\right. \\
& \left.-\frac{T^{2}(B+B q+3-q)}{2\left(1-k^{2}\right)}\right) \\
& \left.+c_{1}^{2}+\left(\frac{(A-B)(1+q) T^{2}}{4\left(1-k^{2}\right)}\right) c_{2}\right] z^{2} \\
& +\left(\frac{(A-B)(1+q) T^{2}}{4\left(1-k^{2}\right)}\right)\left[c_{3}+\left(-\frac{\left(T^{2}-1\right)}{3}\right.\right. \\
& \left.-\frac{T^{2}(B+B q+3-q)}{2\left(1-k^{2}\right)}\right) c_{1} c_{2}+\left(\frac{2}{45}-\frac{T^{2}}{18}+\frac{T^{4}}{90}\right. \\
& -\frac{T^{2}\left(T^{2}-1\right)(B+B q+3-q)}{24\left(1-k^{2}\right)} \\
& +\frac{(B+B q+3-q) T^{2}}{48\left(1-k^{2}\right)^{2}}\left(2 T^{2} k^{2}-3 T^{2} q+7 T^{2}+2\right. \\
& \left.\left.\left.-2 k^{2}+3 T^{2} B+3 T^{2} B q\right)\right) c_{1}^{3}\right] z^{3}+\cdots \\
& +x^{2}
\end{aligned}
$$


If $D_{q} f(z)=1+\sum_{2}^{\infty}[n]_{q} a_{n} z^{n}$ and $f(z)=z+\sum_{2}^{\infty} a_{n} z^{n}$, then

$$
\begin{aligned}
& \frac{z D_{q} f(z)}{f(z)}=\frac{z+\sum_{2}^{\infty}[n]_{q} a_{n} z^{n}}{z+\sum_{2}^{\infty} a_{n} z^{n}} \\
& =1+\left(q a_{2}\right) z+\left[\left(q+q^{2}\right) a_{3}-q a_{2}^{2}\right] z^{2} \\
& \quad+\left[q a_{2}^{3}-a_{2} a_{3}\left(2 q+q^{2}\right)+a_{4}\left(q+q^{2}+q^{3}\right)\right] z^{3} \\
& \quad+\cdots .
\end{aligned}
$$

From (59) and (60), comparison of coefficients of $z$ and $z^{2}$ gives

$$
a_{2}=\frac{(1+q)(A-B) T^{2} c_{1}}{4 q\left(1-k^{2}\right)}
$$

and

$$
\begin{aligned}
a_{3} & =\left(\frac{(1+q) T^{4}(A-B)^{2}}{16 q^{2}\left(1-k^{2}\right)^{2}}+\frac{(A-B) T^{2}\left(T^{2}-1\right)}{24 q\left(1-k^{2}\right)}\right. \\
& \left.-\frac{T^{4}(A-B)}{16 q\left(1-k^{2}\right)^{2}}[(B+1)(1+q)+2(1-q)]\right) c_{1}^{2} \\
& +\frac{T^{2}(A-B)}{4 q\left(1-k^{2}\right)} c_{2} .
\end{aligned}
$$

Now for a real number $\mu$, we consider

$$
\begin{aligned}
& \left|a_{3}-\mu a_{2}^{2}\right|=\mid\left(\frac{(1+q) T^{4}(A-B)^{2}}{16 q^{2}\left(1-k^{2}\right)^{2}}\right. \\
& +\frac{(A-B) T^{2}\left(T^{2}-1\right)}{24 q\left(1-k^{2}\right)} \\
& \left.-\frac{T^{4}(A-B)((B+1)(1+q)+2(1-q))}{16 q\left(1-k^{2}\right)^{2}}\right) c_{1}^{2} \\
& +\left[\frac{T^{2}(A-B)}{4 q\left(1-k^{2}\right)}\right] c_{2}-\mu \frac{(1+q)^{2} T^{4}(A-B)^{2}}{16 q^{2}\left(1-k^{2}\right)^{2}} c_{1}^{2} \mid \\
& +\frac{T^{2}(A-B) \mid}{4 q\left(1-k^{2}\right) \mid} \mid c_{2}-\left(-\frac{(1+q) T^{2}(A-B)}{4 q\left(1-k^{2}\right)}\right. \\
& \left.+\mu \frac{(1+q)^{2} T^{2}(A-B)}{4 q\left(1-k^{2}\right)}\right) c_{1}^{2}\left|=\frac{T^{2}(A-B)}{4 q\left(1-k^{2}\right)}\right| c_{2} \\
& +\frac{\left(T^{2}-1\right)}{6}+\frac{T^{2}((B+1)(1+q)+2(1-q))}{4\left(1-k^{2}\right)}
\end{aligned}
$$

where

$$
\begin{aligned}
v= & \frac{T^{2}}{4\left(1-k^{2}\right)}[(B+1)(1+q)+2(1-q)] \\
& -\frac{\left(T^{2}-1\right)}{6} \\
& -\frac{(1+q) T^{2}(A-B)}{4 q\left(1-k^{2}\right)}(1-\mu(1+q)) .
\end{aligned}
$$

Applying Lemma 4, we get the required result. Inequality (48) is sharp and equality holds for $\mu<\gamma_{1}$ or $\mu>\gamma_{2}$ when $f(z)$ is $f_{1}(z)$ or one of its rotations, where $f_{1}(z)$ is defined such that

$$
\begin{aligned}
& \frac{z D_{q} f_{1}(z)}{f_{1}(z)} \\
& =\frac{(A(1+q)+(3-q)) \tilde{p}_{k}(z)-(A(1+q)-(3-q))}{(B(1+q)+(3-q)) \tilde{p}_{k}(z)-(B(1+q)-(3-q))} .
\end{aligned}
$$

If $\gamma_{1}<\mu<\gamma_{2}$, then, the equality holds for the function $f_{2}(z)$ or one of its rotations, where $f_{2}(z)$ is defined such that

$$
\begin{aligned}
& \frac{z D_{q} f_{2}(z)}{f_{2}(z)} \\
& =\frac{(A(1+q)+(3-q)) \tilde{p}_{k}\left(z^{2}\right)-(A(1+q)-(3-q))}{(B(1+q)+(3-q)) \tilde{p}_{k}\left(z^{2}\right)-(B(1+q)-(3-q))} .
\end{aligned}
$$

If $\mu=\gamma_{1}$, the equality holds for the function $f_{3}(z)$ or one of its rotations, where $f_{3}(z)$ is defined such that

$$
\begin{aligned}
& \frac{z D_{q} f_{3}(z)}{f_{3}(z)}=\left(\frac{1+\eta}{2}\right) \\
& \cdot\left(\frac{(A(1+q)+(3-q)) \tilde{p}_{k}(z)-(A(1+q)-(3-q))}{(B(1+q)+(3-q)) \tilde{p}_{k}(z)-(B(1+q)-(3-q))}\right) \\
& +\left(\frac{1-\eta}{2}\right) \\
& \cdot\left(\frac{(A(1+q)+(3-q)) \tilde{p}_{k}(-z)-(A(1+q)-(3-q))}{(B(1+q)+(3-q)) \tilde{p}_{k}(-z)-(B(1+q)-(3-q))}\right),
\end{aligned}
$$

where $0 \leq \eta \leq 1$. If $\mu=\gamma_{2}$, then, the equality holds for $f(z)$, which is such that $z D_{q} f(z) / f(z)$ is reciprocal of one of the function such that equality holds in the case of $\mu=\gamma_{1}$, where $\gamma_{1}$ and $\gamma_{2}$ are defined by (49) and (50), respectively.

For $k=0$, the above result reduces to the following result, proved by Srivastava et al. [29].

Corollary 11. Let $f \in S_{q}^{*}[A, B],-1 \leq B<A \leq 1$ and be of the form (5). Then for real number $\mu$, we have 


$$
\left|a_{3}-\mu a_{2}^{2}\right| \leq \frac{A-B}{4 q^{2}} \begin{cases}2 q-(B+1)(1+q) q-2(1-q) q+(1+q)(A-B)(1-\mu(1+q)), & \mu \leq \gamma_{3}, \\ 2 q, & \gamma_{3} \leq \mu \leq \gamma_{4} \\ (B+1)(1+q) q+2(1-q) q-2 q-(1+q)(A-B)(1-\mu(1+q)), & \mu \geq \gamma_{4},\end{cases}
$$

where

$$
\gamma_{3}=\frac{1}{(1+q)}-\frac{(B+1)(1+q) q+2(1-q) q}{(1+q)^{2}(A-B)}
$$

$$
+\frac{4 q}{(1+q)^{2}(A-B)}
$$

Inequality (68) is sharp.

and

$$
\gamma_{4}=\frac{1}{(1+q)}-\frac{(B+1)(1+q) q+2(1-q) q}{(1+q)^{2}(A-B)}
$$

For $q \longrightarrow 1^{-}$, Theorem 10 reduces to the following result.

Corollary 12. Let $f \in k-S T[A, B],-1 \leq B<A \leq 1,0<$ $k<1$ and be of the form (5). Then for real number $\mu$, we have

$$
\left|a_{3}-\mu a_{2}^{2}\right| \leq \frac{(A-B) T^{2}}{4\left(1-k^{2}\right)} \begin{cases}\frac{2\left(T^{2}+2\right)}{3}-\frac{2(B+1) T^{2}}{\left(1-k^{2}\right)}+\frac{2 T^{2}(A-B)}{\left(1-k^{2}\right)}(1-2 \mu), & \mu \leq \gamma_{5}, \\ 2, & \gamma_{5} \leq \mu \leq \gamma_{6} \\ \frac{2(B+1) T^{2}}{\left(1-k^{2}\right)}-\frac{2\left(T^{2}+2\right)}{3}-\frac{2 T^{2}(A-B)}{\left(1-k^{2}\right)}(1-2 \mu), & \mu \geq \gamma_{6},\end{cases}
$$

where

$$
\gamma_{5}=\frac{1}{2}+\frac{\left(T^{2}-1\right)\left(1-k^{2}\right)}{6 T^{2}(A-B)}-\frac{(B+1)}{2(A-B)}
$$

$$
+\frac{\left(1-k^{2}\right)}{T^{2}(A-B)}
$$

and

$$
\gamma_{6}=\frac{1}{2}+\frac{\left(T^{2}-1\right)\left(1-k^{2}\right)}{6 T^{2}(A-B)}-\frac{(B+1)}{2(A-B)}
$$

Inequality (71) result is sharp.

Theorem 13. Let $f \in 1-S_{q}[A, B],-1 \leq B \leq A \leq 1$ and be of the form (5). Then for real number $\mu$, we have

$$
\left|a_{3}-\mu a_{2}^{2}\right| \leq \frac{A-B}{q \pi^{2}} \begin{cases}\frac{4}{3}-\frac{4}{\pi^{2}}(2(1-q)+(B+1)(q+1))+\frac{4(A-B)(1+q)}{q \pi^{2}}(1-(q+1) \mu), & \mu \leq \alpha_{1}, \\ 2, & \alpha_{1} \leq \mu \leq \alpha_{2}, \\ -\frac{4}{3}+\frac{4}{\pi^{2}}(2(1-q)+(B+1)(q+1))-\frac{4(A-B)(1+q)}{q \pi^{2}}(1-(1+q) \mu), & \mu \geq \alpha_{2},\end{cases}
$$

where

$$
\begin{aligned}
\alpha_{1}= & \frac{1}{1+q}-\frac{q \pi^{2}}{6(A-B)(1+q)^{2}} \\
& -\frac{2 q(1-q)+q(B+1)(q+1)}{(A-B)(1+q)^{2}}
\end{aligned}
$$

and

$$
\begin{aligned}
\alpha_{2}= & \frac{1}{1+q}+\frac{5 q \pi^{2}}{6(1+q)^{2}(A-B)} \\
& -\frac{2 q(1-q)+q(B+1)(q+1)}{(A-B)(1+q)^{2}} .
\end{aligned}
$$

Inequality (74) is sharp. 
Proof. The use of function $\widetilde{p}(z)=1+\left(2 / \pi^{2}\right)(\log ((1+\sqrt{z}) /(1-$ $\sqrt{z})$ )) instead of

$$
\begin{array}{r}
\tilde{p}_{k}(z)=1 \\
+\frac{2}{1-k^{2}} \sinh ^{2}\left[\left(\frac{2}{\pi} \arccos k\right) \arctan h \sqrt{z}\right], \\
0<k<1,
\end{array}
$$

in the proof of Theorem 10 and following the similar method as followed in Theorem 10 will lead us to the required result.

For $q \longrightarrow 1^{-}$, the theorem reduces to the following form.

Corollary 14. Let $f \in S T[A, B],-1 \leq B<A \leq 1$ and be of the form (5). Then, for a real number $\mu$,

$$
\left|a_{3}-\mu a_{2}^{2}\right| \leq \frac{A-B}{\pi^{2}}\left\{\begin{array}{lr}
\frac{4}{3}-\frac{8}{\pi^{2}}(B+1)+\frac{8}{\pi^{2}}(A-B)(1-2 \mu), & \mu \leq \frac{1}{2}-\frac{\pi^{2}}{24(A-B)}-\frac{B+1}{2(A-B)} \\
& --------- \\
2, & \frac{1}{2}-\frac{\pi^{2}}{24(A-B)}-\frac{B+1}{2(A-B)} \leq \mu \\
& \leq \frac{1}{2}+\frac{5 \pi^{2}}{24(A-B)}-\frac{B+1}{2(A-B)} \\
& -------- \\
-\frac{4}{3}+\frac{8}{\pi^{2}}(B+1)-\frac{8}{\pi^{2}}(A-B)(1-2 \mu), & \mu \geq \frac{1}{2}+\frac{5 \pi^{2}}{24(A-B)}-\frac{B+1}{2(A-B)} .
\end{array}\right.
$$

This result is sharp.

Now we consider the inverse function $\mathscr{F}$, defined as $\mathscr{F}(w)=\mathscr{F}(f(z))=z, z \in \mathcal{U}$, and we find the following coefficient bound for inverse functions.

Theorem 15. Let $w=f(z) \in k-S T_{q}[A, B],-1 \leq B<A \leq$ $1,0<k<1$, and $\mathscr{F}(w)=f^{-1}(w)=w+\sum_{n=2}^{\infty} d_{n} w^{n}$. Then,

$$
\left|d_{n}\right| \leq \frac{(A-B)(1+q) T^{2}}{2 q\left(1-k^{2}\right) \sum_{k=0}^{n-2} q^{k}} \quad(n=2,3,4) .
$$

Proof. Since $\mathscr{F}(w)=\mathscr{F}(f(z))=z$, it is easy to see that

$$
\begin{aligned}
& d_{2}=-a_{2}, \\
& d_{3}=2 a_{2}^{2}-a_{3}, \\
& d_{4}=-a_{4}+5 a_{2} a_{3}-5 a_{2}^{3} .
\end{aligned}
$$

By using (61) and (62), one can have

$$
d_{2}=\frac{-(1+q)(A-B) T^{2} c_{1}}{4 q\left(1-k^{2}\right)}
$$

and

$$
\begin{aligned}
d_{3} & =\left(\frac{(A-B)^{2}(1+q)^{2} T^{4}}{8 q^{2}\left(1-k^{2}\right)^{2}}-\frac{(A-B)^{2}(1+q) T^{4}}{16 q^{2}\left(1-k^{2}\right)^{2}}\right. \\
& -\frac{(A-B) T^{2}\left(T^{2}-1\right)}{24 q\left(1-k^{2}\right)} \\
& \left.+\frac{(A-B) T^{4}(B+B q+3-q)}{16 q\left(1-k^{2}\right)^{2}}\right) c_{1}^{2} \\
& +\left(-\frac{(A-B) T^{2}}{4 q\left(1-k^{2}\right)}\right) c_{2} .
\end{aligned}
$$

From (59) and (60), comparison of $z^{3}$ gives

$$
\begin{aligned}
d_{4} & =\left(-\frac{(A-B)(1+q) T^{2}}{4\left(q+q^{2}+q^{3}\right)\left(1-k^{2}\right)}\right)\left\{c_{3}+\left(\frac{T^{2}}{3}\right.\right. \\
& -\frac{(A-B)\left(5 q^{2}+4 q+3\right) T^{2}}{4 q\left(1-k^{2}\right)}-\frac{1}{3} \\
& \left.-\frac{T^{2}(B+B q+3-q)}{2\left(1-k^{2}\right)}\right) c_{2} c_{1} \\
& +\left(\frac{(A-B)^{2}(1+q)\left(5 q^{3}+5 q^{2}+5 q+1\right) T^{4}}{16 q^{2}\left(1-k^{2}\right)^{2}}\right. \\
& -\frac{(A-B)\left(5 q^{2}+4 q+3\right) T^{2}\left(T^{2}-1\right)}{24 q\left(1-k^{2}\right)^{2}} \\
& +\frac{(A-B)\left(5 q^{2}+4 q+3\right) T^{4}(B+B q+3-q)}{16 q\left(1-k^{2}\right)^{2}} \\
& -\frac{T^{2}\left(T^{2}-1\right)(B+B q+3-q)}{24\left(1-k^{2}\right)}+\frac{2}{45}-\frac{T^{2}}{18}+\frac{T^{4}}{90} \\
& +\frac{T^{2}(B+B q+3-q)}{48\left(1-k^{2}\right)^{2}}\left(2 T^{2} k^{2}-3 T^{2} q+7 T^{2}+2\right. \\
- & \left.\left.\left.2 k^{2}+3 T^{2} B+3 T^{2} B q\right)\right) c_{1}^{3}\right\} .
\end{aligned}
$$

Now, from (81) and (82), one can have

$$
\left|d_{2}\right| \leq \frac{(A-B)(1+q) T^{2}}{2 q\left(1-k^{2}\right)}
$$


and

$$
\left|d_{3}\right| \leq \frac{(A-B) T^{2}}{4 q\left(1-k^{2}\right)}\left\{\left|\lambda_{1}\right|\left|c_{2}-c_{1}^{2}\right|+\left|\lambda_{2}\right|\left|c_{2}\right|\right\}
$$

where

$$
\begin{aligned}
\lambda_{1}= & \frac{1}{6}+\frac{(A-B)(1+q)^{2} T^{2}}{2 q\left(1-k^{2}\right)}-\frac{(1+q) T^{2}(A-B)}{4 q\left(1-k^{2}\right)} \\
& -\frac{T^{2}}{6}+\frac{T^{2}(B+B q+3-q)}{4\left(1-k^{2}\right)}
\end{aligned}
$$

and

$$
\begin{aligned}
\lambda_{2}= & \frac{5}{6}-\frac{(A-B)(1+q)^{2} T^{2}}{2 q\left(1-k^{2}\right)}+\frac{(1+q) T^{2}(A-B)}{4 q\left(1-k^{2}\right)} \\
& +\frac{T^{2}}{6}-\frac{T^{2}(B+B q+3-q)}{4\left(1-k^{2}\right)} .
\end{aligned}
$$

Application of the bounds $\left|c_{2}-c_{1}^{2}\right| \leq 2$ and $\left|c_{2}\right| \leq 2$ (see Lemma 4 for $v=1$ and $v=0)$ gives $\left|d_{3}\right| \leq(A-B) T^{2} / 2 q(1-$ $k^{2}$ ). Lastly, (83) reduces to

$$
\begin{aligned}
& \left|d_{4}\right| \leq \frac{(A-B)(1+q) T^{2}}{4\left(q+q^{2}+q^{3}\right)\left(1-k^{2}\right)}\left[\left|\lambda_{3}\right|\left|c_{3}-2 c_{2} c_{1}+c_{1}^{3}\right|\right. \\
& \left.\quad+\left|\lambda_{4}\right|\left|c_{3}-c_{2} c_{1}\right|+\left|\lambda_{5}\right|\left|c_{3}\right|\right]
\end{aligned}
$$

where

$$
\begin{aligned}
\lambda_{3} & =\frac{2}{45}+\frac{T^{2}}{6}\left(-\frac{1}{3}+\frac{(A-B)\left(5 q^{2}+4 q+3\right)}{4 q\left(1-k^{2}\right)^{2}}\right. \\
& \left.+\frac{(B+B q+3-q)}{2\left(1-k^{2}\right)}\right) \\
& +T^{4}\left(\frac{(A-B)^{2}(1+q)\left(5 q^{3}+5 q^{2}+5 q+1\right)}{16 q^{2}\left(1-k^{2}\right)^{2}}\right. \\
& -\frac{(A-B)\left(5 q^{2}+4 q+3\right)}{24 q\left(1-k^{2}\right)^{2}} \\
& +\frac{(A-B)\left(5 q^{2}+4 q+3\right)(B+B q+3-q)}{16 q\left(1-k^{2}\right)^{2}} \\
& \left.-\frac{(B+B q+3-q)}{24\left(1-k^{2}\right)}+\frac{1}{90}\right)+\frac{T^{2}(B+B q+3-q)}{24\left(1-k^{2}\right)}(-1
\end{aligned}
$$

$$
\begin{aligned}
+ & \left.\frac{\left(2 T^{2} k^{2}-3 T^{2} q+7 T^{2}+2-2 k^{2}+3 T^{2} B+3 T^{2} B q\right)}{2\left(1-k^{2}\right)}\right) \\
\lambda_{4} & =\frac{22}{90}+\frac{2 T^{2}}{3}\left(-\frac{1}{3}+\frac{(A-B)\left(5 q^{2}+4 q+3\right)}{4 q\left(1-k^{2}\right)^{2}}\right. \\
+ & \left.\frac{(B+B q+3-q)}{2\left(1-k^{2}\right)}\right) \\
- & 2 T^{4}\left(\frac{(A-B)^{2}(1+q)\left(5 q^{3}+5 q^{2}+5 q+1\right)}{16 q^{2}\left(1-k^{2}\right)^{2}}\right. \\
& -\frac{(A-B)\left(5 q^{2}+4 q+3\right)}{24 q\left(1-k^{2}\right)^{2}} \\
+ & \frac{(A-B)\left(5 q^{2}+4 q+3\right)(B+B q+3-q)}{16 q\left(1-k^{2}\right)^{2}} \\
& \left.+\frac{(B+B q+3-q)}{24\left(1-k^{2}\right)}+\frac{1}{90}\right)-\frac{T^{2}(B+B q+3-q)}{12\left(1-k^{2}\right)}(-1 \\
+ & \left.\frac{\left(2 T^{2} k^{2}-3 T^{2} q+7 T^{2}+2-2 k^{2}+3 T^{2} B+3 T^{2} B q\right)}{2\left(1-k^{2}\right)}\right)
\end{aligned}
$$

and

$$
\begin{aligned}
\lambda_{5} & =\frac{32}{45}-\frac{5 T^{2}}{6}\left(-\frac{1}{3}+\frac{(A-B)\left(5 q^{2}+4 q+3\right)}{4 q\left(1-k^{2}\right)^{2}}\right. \\
& \left.+\frac{(B+B q+3-q)}{2\left(1-k^{2}\right)}\right) \\
& +T^{4}\left(\frac{(A-B)^{2}(1+q)\left(5 q^{3}+5 q^{2}+5 q+1\right)}{16 q^{2}\left(1-k^{2}\right)^{2}}\right. \\
& -\frac{(A-B)\left(5 q^{2}+4 q+3\right)}{24 q\left(1-k^{2}\right)^{2}} \\
& +\frac{(A-B)\left(5 q^{2}+4 q+3\right)(B+B q+3-q)}{16 q\left(1-k^{2}\right)^{2}} \\
& \left.+\frac{(B+B q+3-q)}{24\left(1-k^{2}\right)}+\frac{1}{90}\right)+\frac{T^{2}(B+B q+3-q)}{24\left(1-k^{2}\right)}(-1 \\
& \left.+\frac{\left(2 T^{2} k^{2}-3 T^{2} q+7 T^{2}+2-2 k^{2}+3 T^{2} B+3 T^{2} B q\right)}{2\left(1-k^{2}\right)}\right) .
\end{aligned}
$$

Applying the bounds $\left|c_{3}-2 c_{2} c_{1}+c_{1}^{3}\right| \leq 2$, see [32], $\left|c_{3}-c_{2} c_{1}\right| \leq 2$ and $\left|c_{3}\right| \leq 2$, see [31], to the right hand side of (88) and using the fact that $\lambda_{i} \geq 0, i=1,2,3$, we have $\left|d_{4}\right| \leq(A-B)(1+$ q) $T^{2} / 2\left(1-k^{2}\right)\left(q+q^{2}+q^{3}\right)$ and this completes the proof. 
Theorem 16. Let $w=f(z) \in k-S T_{q}[A, B],-1 \leq B<A \leq$ $1,0<k<1$, and $\mathscr{F}(w)=f^{-1}(w)=w+\sum_{n=2}^{\infty} d_{n} w^{n}$. Then for real number $\mu$, we have

$$
\begin{aligned}
& \left|d_{3}-\mu d_{2}^{2}\right| \\
& \leq \frac{(A-B) T^{2}}{4 q\left(1-k^{2}\right)} \begin{cases}\frac{4}{3}+\frac{2 T^{2}}{3}-\frac{T^{2}(B+B q+3-q)}{\left(1-k^{2}\right)}-\frac{T^{2}(A-B)(q+1)}{q\left(1-k^{2}\right)}((1+2 q)-\mu(1+q)), & \mu \leq \alpha_{3}, \\
2, & \alpha_{3} \leq \mu \leq \alpha_{4}, \\
-\frac{4}{3}-\frac{2 T^{2}}{3}+\frac{T^{2}(B+B q+3-q)}{\left(1-k^{2}\right)}+\frac{T^{2}(A-B)(q+1)}{q\left(1-k^{2}\right)}((1+2 q)-\mu(1+q)), & \mu \geq \alpha_{4},\end{cases}
\end{aligned}
$$

where

$$
\begin{aligned}
\alpha_{3}= & \frac{1+2 q}{1+q}+\frac{2 q\left(1-k^{2}\right)}{3(1+q)^{2}(A-B) T^{2}} \\
& -\frac{2 q\left(1-k^{2}\right)}{3(1+q)^{2}(A-B)}+\frac{q(B+B q+3-q)}{(1+q)^{2}(A-B)}
\end{aligned}
$$

Proof. The proof follows directly from (81), (82), and Lemma 4.

Following the similar method as proceeded in Theorems 15 and 16, we have the following results for $k=1$.

Theorem 17. Let $w=f(z) \in 1-S T_{q}[A, B],-1 \leq B<A \leq 1$, and $\mathscr{F}(w)=f^{-1}(w)=w+\sum_{n=2}^{\infty} M_{n} w^{n}$. Then,

$$
\left|M_{n}\right| \leq \frac{2(A-B)(q+1)}{q \pi^{2} \sum_{k=0}^{n-2} q^{k}} \quad(n=2,3,4) .
$$

Theorem 18. Let $w=f(z) \in 1-S T_{q}[A, B],-1 \leq B<A \leq 1$, and $\mathscr{F}(w)=f^{-1}(w)=w+\sum_{n=2}^{\infty} M_{n} w^{n}$. Then for real number $\mu$, we have

$$
\left|M_{3}-\mu M_{2}^{2}\right| \leq \frac{A-B}{q \pi^{2}} \begin{cases}\frac{4}{3}-\frac{4(q+1)(B+B q+3-q)}{\pi^{2}}-\frac{4(A-B)}{q \pi^{2}}\left(\left(2 q^{2}+3 q+1\right)^{2}-(1+q)^{2} \mu\right), & \mu \leq \alpha_{5}, \\ 2, & \alpha_{5} \leq \mu \leq \alpha_{6}, \\ -\frac{4}{3}+\frac{4(q+1)(B+B q+3-q)}{\pi^{2}}+\frac{4(A-B)}{q \pi^{2}}\left(\left(2 q^{2}+3 q+1\right)^{2}-(1+q)^{2} \mu\right), & \mu \geq \alpha_{6} .\end{cases}
$$

where

$$
\begin{aligned}
\alpha_{5}= & \frac{\left(2 q^{2}+3 q+1\right)}{(1+q)^{2}}+\frac{q \pi^{2}}{6(A-B)(q+1)^{2}} \\
& +\frac{q(B+B q+3-q)}{(A-B)(q+1)}
\end{aligned}
$$

and

$$
\begin{aligned}
\alpha_{6}= & \frac{\left(2 q^{2}+3 q+1\right)}{(1+q)^{2}}-\frac{5 q \pi^{2}}{6(A-B)(q+1)^{2}} \\
& +\frac{q(B+B q+3-q)}{(A-B)(q+1)} .
\end{aligned}
$$

\section{Conclusion}

We have generalized the concept of q-starlike functions by associating them with conic domain. The concept of qderivatives is used to define class of certain analytic functions. The certain renowned coefficient inequalities are found for these functions and their inverse functions.

\section{Data Availability}

No data were used to support this study.

\section{Conflicts of Interest}

The authors declare that there are no conflicts of interest regarding the publication of this article. 


\section{Authors' Contributions}

All authors contributed equally to and approved the final manuscript.

\section{Acknowledgments}

This research is partially supported by Sarhad University of Science and IT, Ring Road, Peshawar, Pakistan. The authors are grateful to their institutions' heads for the conducive research environment.

\section{References}

[1] H. Al dweby and M. Darus, "On Harmonic Meromorphic Functions Associated with Basic Hypergeometric Functions," The Scientific World Journal, vol. 2013, Article ID 164287, 7 pages, 2013.

[2] H. Aldweby and M. Darus, "A subclass of harmonic univalent functions associated with q-analogue of Dziok-Serivastva operator," ISRN Mathematical Analysis, vol. 2013, Article ID 382312, 6 pages, 2013.

[3] S. Altinkaya and S. Yalcin, "On the Fekete-Szegö problem for analytic functions defined by using symmetric q-derivative operator," Konuralp J. Maths, vol. 5, no. 1, pp. 176-186, 2017.

[4] G. A. Anastassiou and S. G. Gal, "Geometric and approximation properties of some singular integrals in the unit disk," Journal of Inequalities and Applications, vol. 2006, Article ID 17231, 19 pages, 2006.

[5] G. A. Anastassiou and S. G. Gal, "Geometric and approximation properties of generalized singular integrals in the unit disk," Journal of the Korean Mathematical Society, vol. 43, no. 2, pp. 425-443, 2006.

[6] A. Aral and V. Gupta, "On q-Baskakov type operators," Demonstratio Mathematica, vol. 42, no. 1, pp. 109-122, 2009.

[7] A. Aral and V. Gupta, "On the Durrmeyer type modification of the q-Baskakov type operators," Nonlinear Analysis: Theory, Methods \& Applications, vol. 72, no. 3-4, pp. 1171-1180, 2010.

[8] A. Aral and V. Gupta, "Generalized q-Baskakov operators," Mathematica Slovaca, vol. 61, no. 4, pp. 619-634, 2011.

[9] S. Bulut, "Certain subclasses of analytic and bi-univalent functions involving the q-derivative operator," Commun. Fac. Sci. Univ. Ank. Sér. A1 Math. Stat, vol. 66, no. 1, pp. 108-114, 2017.

[10] D. V. Dolgy, D. S. Kim, T. Kim, and S.-H. Rim, "Some identities of special q-polynomials," Journal of Inequalities and Applications, 2014:438, 10 pages, 2014.

[11] H. G. Griffin, "Attenuated Salmonella as live vaccines: prospects for multivalent poultry vaccines," World's Poultry Science Journal, vol. 47, pp. 129-140, 1991.

[12] M. E. Ismail, E. Merkes, and D. Styer, "A generalization of starlike functions," Complex Variables, Theory and Application, vol. 14, no. 1-4, pp. 77-84, 1990.

[13] F. H. Jackson, "On q-functions and a certain difference operator," Earth and Environmental Science Transactions of the Royal Society of Edinburgh, vol. 46, no. 2, pp. 253-281, 1908.

[14] F. H. Jackson, "On q-definite integrals," Quarterly Journal of Pure and Applied Mathematics, vol. 41, pp. 193-203, 1910.

[15] H. M. Srivastava, "Some generalizations and basic (or q-) extensions of the Bernoulli, Euler and Genocchi polynomials," Applied Mathematics \& Information Sciences, vol. 5, no. 3, pp. 390-444, 2011.
[16] S. Agrawal and S. K. Sahoo, "On a generalization of close-toconvex functions," Annales Polonici Mathematici, vol. 113, no. 1, pp. 93-108, 2015.

[17] M. A. Noor, K. I. Noor, and S. Iftikhar, "Integral inequalities for differentiable relative harmonic preinvex functions (survey)," TWMS Journal of Pure and Applied Mathematics, vol. 7, no. 1, pp. 3-19, 2016.

[18] K. I. Noor, S. Riaz, and M. A. Noor, "On q-bernardi integral operator," TWMS Journal of Pure and Applied Mathematics, vol. 8, no. 1, pp. 3-11, 2017.

[19] C. Ramachandran, T. Soupramanien, and B. A. Frasin, "New subclasses of analytic function associated with q-difference operator," European Journal of Pure and Applied Mathematics, vol. 10, no. 2, pp. 348-362, 2017.

[20] S. Mahmood and J. Sokół, "New subclass of analytic functions in conical domain associated with Ruscheweyh q-differential operator," Results in Maths, vol. 71, no. 3, pp. 1345-1357, 2017.

[21] S. Kanas and A. Wisniowska, "Conic regions and k-uniform convexity," Journal of Computational and Applied Mathematics, vol. 105, no. 1-2, pp. 327-336, 1999.

[22] S. A. Kanas and A. Wisniowska, "Conic domains and starlike functions," Revue Roumaine de Mathématique Pures et Appliquées, vol. 45, no. 4, pp. 647-657, 2000.

[23] S. Kanas and H. M. Srivastava, "Linear operators associated with k-uniformly convex functions," Integral Transforms and Special Function, vol. 9, no. 2, pp. 121-132, 2000.

[24] S. Mahmood, S. N. Malik, S. Farman, S. M. J. Riaz, and S. Farwa, "Uniformly Alpha-Quasi-Convex Functions Defined by Janowski Functions," Journal of Function Spaces, vol. 2018, Article ID 6049512, 7 pages, 2018.

[25] S. Mahmood, S. N. Malik, S. Mustafa, and S. M. J. Riaz, "A new Subclass of kJanowski Type Functions Associated with Ruscheweyh Derivative," Journal of Function Spaces, vol. 2017, Article ID 6095293, 2017.

[26] S. Kanas, "Coefficient estimates in subclasses of the Caratheodory class related to conical domains," Acta Mathematica Universitatis Comenianae, vol. 74, no. 2, pp. 149-161, 2005.

[27] K. I. Noor and S. N. Malik, "On coefficient inequalities of functions associated with conic domains," Computers \& Mathematics with Applications, vol. 62, no. 5, pp. 2209-2217, 2011.

[28] W. Janowski, "Some extremal problems for certain families of analytic functions," Annales Polonici Mathematici, vol. 28, pp. 297-326, 1973.

[29] H. M. Srivastava, B. Khan, N. Khan, and Q. Zahoor, "Coefficients inequalities for q-starlike functions associated with Janowski functions," Tech. Rep., 2017.

[30] W. Rogosinski, "On the coefficients of subordinate functions," Proceedings of the London Mathematical Society, vol. 48, no. 1, pp. 48-82, 1943.

[31] W. C. Ma and D. Minda, "A unified treatment of some special classes of univalent functions," in Proceedings of the Conference on Complex Analysis (Tianjin '92), pp. 157-169, Internat. Press, Cambridge, MA, USA.

[32] R. J. Libera and E. J. Złotkiewicz, "Early coefficients of the inverse of a regular convex function," Proceedings of the American Mathematical Society, vol. 85, no. 2, pp. 225-230, 1982. 


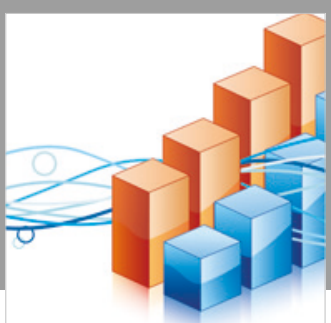

Advances in

Operations Research

\section{-n-m}
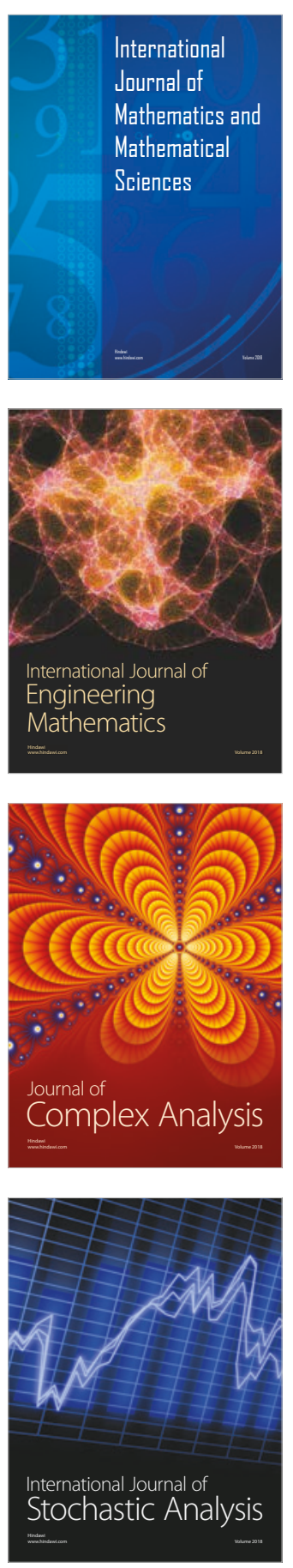
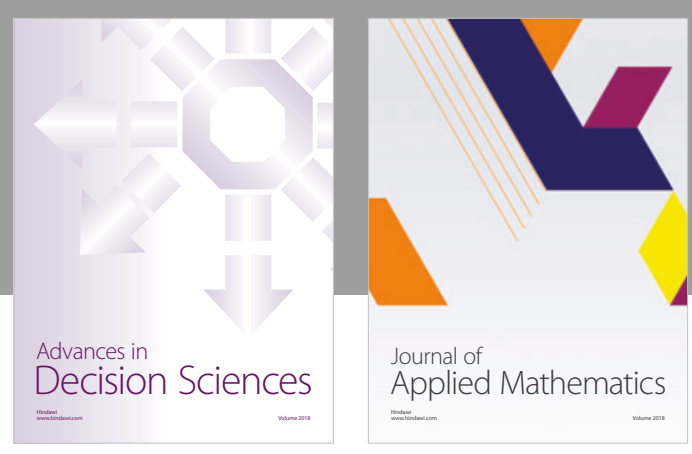

Journal of

Applied Mathematics
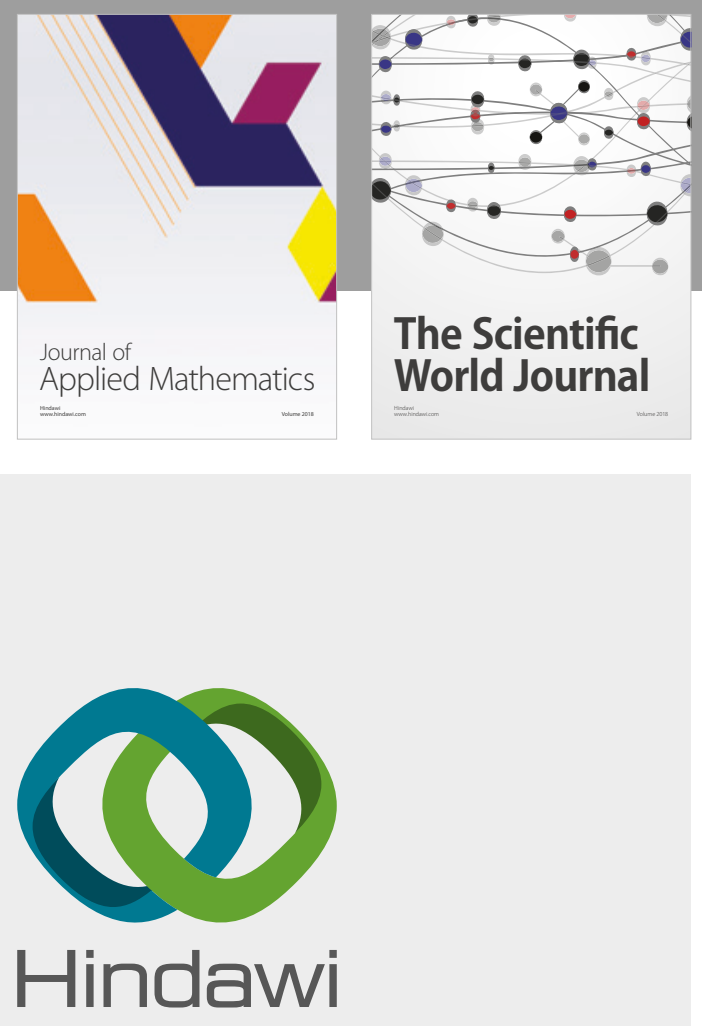

Submit your manuscripts at

www.hindawi.com

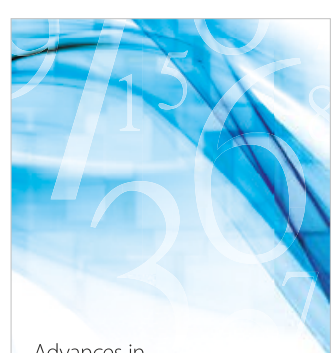

Advances in
Numerical Analysis
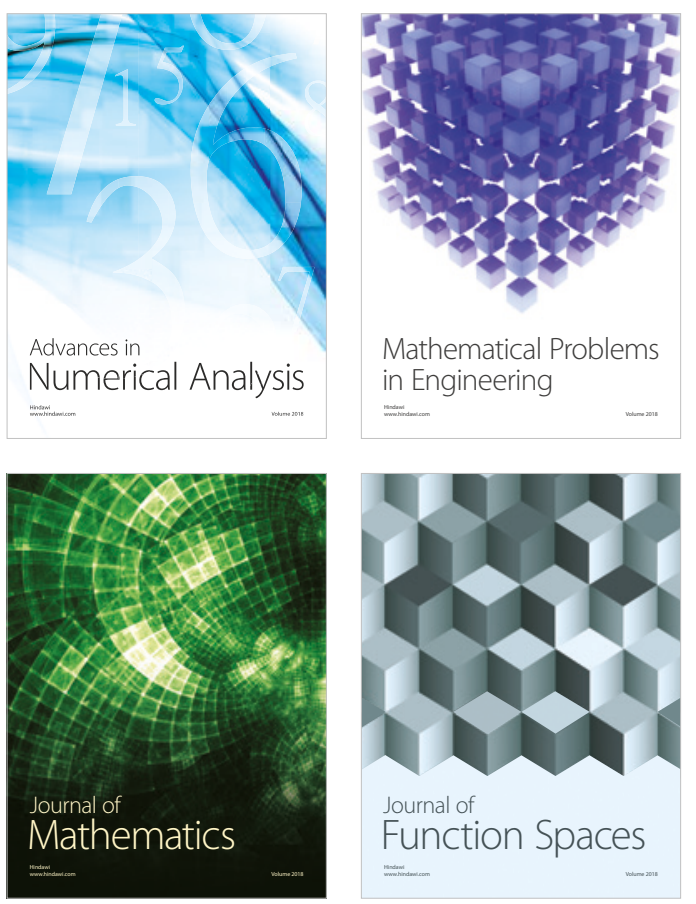

Mathematical Problems in Engineering

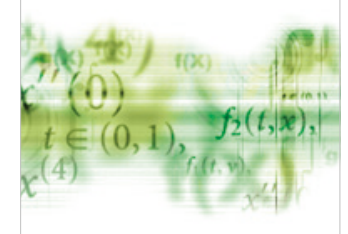

International Journal of

Differential Equations

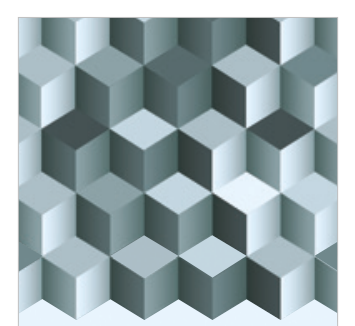

Journal of

Function Spaces

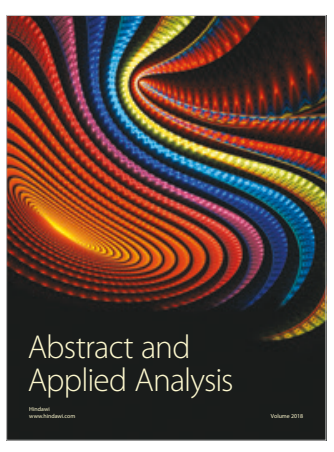

The Scientific

World Journal

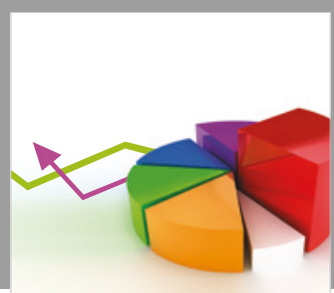

Journal of

Probability and Statistics
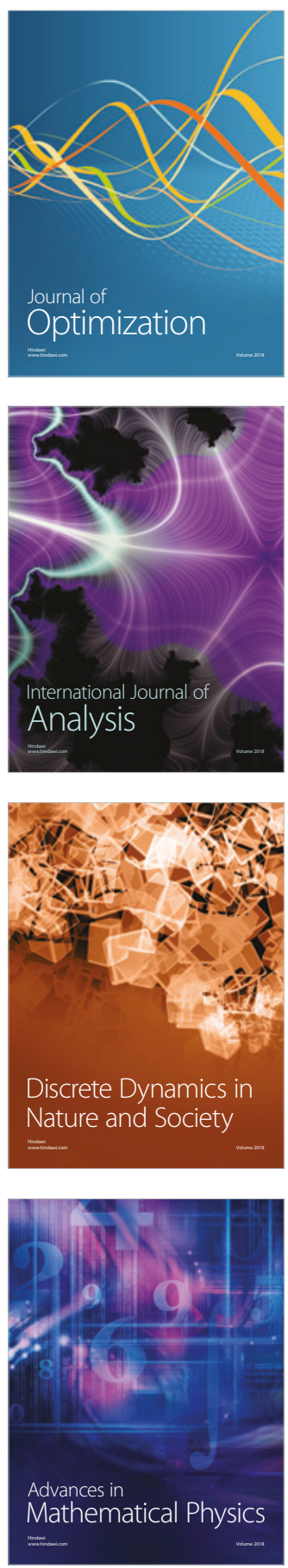